\title{
Tannerella forsythia invasion in oral epithelial cells requires phosphoinositide 3-kinase activation and clathrin-mediated endocytosis
}

Correspondence

Ashu Sharma

sharmaa@buffalo.edu

Received 10 February 2011

Revised 20 April 2011

Accepted 24 May 2011

\section{Elina Mishima and Ashu Sharma}

\author{
Department of Oral Biology, School of Dental Medicine, University at Buffalo, State University of \\ New York, NY, USA
}

\begin{abstract}
Tannerella forsythia, a Gram-negative anaerobe implicated in periodontitis, has been detected within human buccal epithelial cells and shown to invade oral epithelial cells in vitro. We have previously shown that this bacterium triggers host tyrosine kinase-dependent phosphorylation and actin-dependent cytoskeleton reorganization for invasion. On the bacterial side, the leucine-rich repeat cell-surface BspA protein is important for entry. The present study was undertaken to identify host signalling molecules during $T$. forsythia entry into human oral and cervical epithelial cells. Specifically, the roles of phosphatidylinositol 3-kinase (PI3K), Rho-family GTPases, cholesterol-rich membrane microdomains and the endocytic protein clathrin were investigated. For this purpose, cell lines were pretreated with chemical inhibitors or small interfering RNAs (siRNAs) that target PI3Ks, Rho GTPases, clathrin and cholesterol (a critical component of 'lipid rafts'), and the resulting effects on $T$. forsythia uptake were determined. Our studies revealed that T. forsythia entry is dependent on host PI3K signalling, and that purified BspA protein causes activation of this lipid kinase. Bacterial entry also requires the cooperation of host Rac1 GTPase. Finally, our findings indicate an important role for clathrin and cholesterol-rich lipid microdomains in the internalization process
\end{abstract}

\section{INTRODUCTION}

Tannerella forsythia is a Gram-negative oral anaerobe implicated in the development of periodontal disease, an inflammatory disease of the tooth-supporting tissues that results in tooth loss (Tanner \& Izard, 2006). While the contribution of T. forsythia in periodontitis has been well established through epidemiological and clinical intervention studies, the mechanisms underlying $T$. forsythia virulence are poorly understood (Sharma, 2010). T. forsythia has been detected intracellularly in buccal and crevicular epithelium of patients with periodontitis (Colombo et al., 2007; Rudney et al., 2005). Furthermore, T. forsythia has been shown to invade epithelial cells in vitro (Han et al., 2000; Inagaki et al., 2006; Kirschbaum et al., 2010; Sabet et al., 2003). Thus, it is likely that this bacterium exploits epithelial cell invasion to avoid host defence surveillance. In addition, the buccal and crevicular

Abbreviations: Cav-1, caveolin-1; CLTC, clathrin heavy chain; EGF, epidermal growth factor; HRP, horseradish peroxidase; LRR, leucine-rich repeat; $\mathrm{M} \beta \mathrm{CD}$, methyl- $\beta$-cyclodextrin; $\mathrm{MDC}$, monodansylcadaverine; $\mathrm{PI} 3 \mathrm{~K}$, phosphatidylinositol 3-kinase; qRT-PCR, quantitative real-time RT-PCR; RNAi, RNA interference; siRNA, small interfering RNA.

A supplementary figure, showing that C3 toxin disrupts actin stress fibres, is available with the online version of this paper. epithelia may serve as bacterial reservoirs for recurrent infections.

We have previously shown that the T. forsythia leucine-rich repeat (LRR) cell-surface and secreted BspA protein is required for attachment to and invasion of epithelial cells (Inagaki et al., 2006). Moreover, our study demonstrated that this invasion depends on protein phosphorylation and cytoskeleton remodelling (Inagaki et al., 2006). Recently, we demonstrated that the $\mathrm{NanH}$ sialidase expressed by $T$. forsythia promotes bacterial invasion of oral epithelial cells by hydrolysing cell-surface sialic acid residues (Honma et al., 2011). These findings indicate that siliac acid residues mask the epithelial cell epitopes involved in bacterial interactions. Currently, specific receptors and intracellular proteins involved in the entry of $T$. forsythia into epithelial cells have not been identified. In this regard, pathogens are known to usurp host cellular machinery for entry. A majority of bacteria that invade non-phagocytic cells express surface proteins that interact with cellular receptors to initiate signalling cascades that result in membrane 'zippering' for entry (Cossart \& Sansonetti, 2004; Veiga \& Cossart, 2006). Alternatively, bacteria that possess a type III secretion system directly inject protein effectors into the host cytosol, avoiding initial intimate contact with the host cell. These effectors trigger massive actin polymerization and the formation of macropinocytic membrane extensions that 
lead to bacterial internalization ('trigger' mechanisms) (Cossart \& Sansonetti, 2004; Veiga \& Cossart, 2006). With regard to $T$. forsythia, the genome sequence (available at http://www.oralgen.lanl.gov) does not predict the presence of a conventional type III secretion system in the bacterium. The objective of our study was to better understand the molecular mechanism of $T$. forsythia internalization by identifying specific human host proteins that promote bacterial entry.

\section{METHODS}

Chemicals. Genistein, wortmannin, LY294002, chlorpromazine, methyl- $\beta$-cyclodextrin (M $\beta \mathrm{CD})$, monodansylcadaverine (MDC) and nystatin were purchased from Sigma. Cell-permeable C3 transferase from Clostridium botulinum was bought from Cytoskeleton, and EHT1864 was bought from Tocris Bioscience. Stock solutions of each of these chemicals were made in manufacturer-recommended diluents (water or methanol), and serial dilutions of the stock solutions were made in Dulbecco's modified Eagle's medium (DMEM).

Bacterial strains and culture conditions. Porphyromonas gingivalis strain 381 was cultured in trypticase soy broth (BD) supplemented with $0.5 \%$ yeast extract, $0.1 \%$ L-cysteine, $5 \mu \mathrm{g}$ haemin $\mathrm{ml}^{-1}$ and $0.5 \mu \mathrm{g}$ vitamin $\mathrm{K} \mathrm{ml}^{-1}$. T. forsythia ATCC 43037 was cultured as described by Honma et al. (2001). KB cells (CCL-17, ATCC) used as host cells for invasion studies were maintained in DMEM (Invitrogen) supplemented with $10 \%$ fetal bovine serum (Invitrogen). KB cells were originally thought to be derived from an epidermal carcinoma of the mouth, but have subsequently been found to have been established via HeLa cell contamination. The OBA-9 cell line (a gift from D. Demuth, University of Louisville, KY, USA) is a human gingival epithelial cell line immortalized by simian virus transformation. OBA-9 cells were maintained in keratinocyte basal medium KGM-2 supplemented with epidermal growth factor (EGF), bovine pituitary extract, epinephrine, transferrin, hydrocortisone and insulin, as per the manufacturer's recommendations (Lonza). Both cell lines were cultured at $37{ }^{\circ} \mathrm{C}$ under $5 \% \mathrm{CO}_{2}$.

Small interfering RNA (siRNA) transfection. KB cells were seeded at $1.4 \times 10^{4}$ cells per well in 48 -well plates the day prior to transfection. Caveolin-1 (Cav-1), clathrin heavy chain (CLTC), CDC42, Rac1, RhoA and control siRNAs were purchased from Dharmacon. Prior to transfection, we added 90 pmol siRNA with $1.5 \mu \mathrm{l}$ Oligofectamine Reagent (Invitrogen) and Opti-MEM (Invitrogen), followed by $20 \mathrm{~min}$ incubation at room temperature. The KB cells were treated with Oligofectamine-siRNA complex and incubated for $48 \mathrm{~h}$ at $37{ }^{\circ} \mathrm{C}$ under $5 \% \mathrm{CO}_{2}$.

Invasion assay. One day before the invasion assay, KB or OBA-9 cells were seeded at a density of $1 \times 10^{5}$ cells per well in 48 -well plates. Overnight-grown monolayers were treated with inhibitors diluted in plain DMEM for $30 \mathrm{~min}$. T. forsythia cells were added at an m.o.i. of 200. The monolayers were incubated for $4 \mathrm{~h}$, washed with DMEM for $\mathrm{KB}$ cells or keratinocyte basal medium KGM-2 for OBA-9 cells, and then treated with gentamicin $\left(50 \mu \mathrm{g} \mathrm{ml}^{-1}\right)$ and metronidazole $\left(200 \mu \mathrm{g} \mathrm{ml}^{-1}\right)$ to kill extracellular bacteria. The monolayers were washed gently with DMEM or KGM-2, and finally lysed with distilled water to obtain intracellular bacteria for counting.

Quantitative real-time RT-PCR (qRT-PCR). All procedures were according to the manufacturer's protocol. RNA from transfected cells was extracted with an RNeasy kit (Qiagen), followed by treatment with DNase I (Qiagen) to remove residual DNA. cDNA was then generated by using an iScript cDNA Synthesis kit (Bio-Rad). Reactions were set up for qRT-PCR containing iQ SYBR Green SuperMix (Bio-Rad). Expression ratios were calculated according to the $2^{\Delta \Delta \mathrm{C}_{\mathrm{T}}}$ method for each transcript.

Western blotting. To assess phosphatidylinositol 3-kinase (PI3K) activation, epithelial cells were grown in six-well culture plates to approximately $80 \%$ confluence. Cells were then incubated for up to $16 \mathrm{~h}$ in serum-free DMEM (for KB cells) or growth factor-free KGM-2 medium (for OBA-9 cells) before stimulating with either bacteria (m.o.i. 100), BspA $\left(10 \mu \mathrm{g} \mathrm{ml}^{-1}\right)$ or EGF $\left(20 \mathrm{ng} \mathrm{ml}^{-1}\right)$ for 5,15 and $30 \mathrm{~min}$, or left untreated $(0 \mathrm{~min})$. The plates were immediately chilled on ice, and cells were washed with cold PBS and harvested with cold cell lysis buffer (50 mM Tris, $\mathrm{pH}$ 7.4, $0.25 \%$ deoxycholic acid, $1 \%$ Triton $\mathrm{X}-100,1 \mathrm{mM}$ sodium orthovanadate, $1 \mathrm{mM}$ sodium fluoride) containing $1 \mathrm{mM}$ PMSF and protease inhibitor cocktail (Sigma). Lysates were centrifuged at $13000 \mathrm{~g}$ for $10 \mathrm{~min}$, supernatants were collected, and protein concentrations were determined by Pierce BCA Protein Assay reagent (Thermo Scientific). Lysates were separated on $10 \%$ denaturing SDS polyacrylamide gels and electroblotted onto nitrocellulose membranes. Membranes were processed for the detection of target proteins by immunoblotting as per standard protocols. Akt phosphorylated at Ser 473 was detected using rabbit anti-phospho-Akt antibody (Cell Signaling Technology), and total Akt was detected by using rabbit anti-Akt antibody (Cell Signaling Technology). Goat antirabbit horseradish peroxidase (HRP)-conjugated secondary antibody and chemiluminescent membrane peroxidase substrate reagent (KPL) were used for detection. Chemiluminescent bands were detected with a Fuji LAS-1000 Plus imager.

The expression of caveolin-1 (Cav-1) and CLTC was detected by rabbit anti-caveolin and mouse anti-CLTC antibody (BD). As secondary antibodies, goat anti-rabbit HRP-conjugated and goat anti-mouse HRP-conjugated antibodies were used. The protein bands were visualized by the TMB Membrane Peroxidase Substrate System C-3 (KPL).

Data analysis. Data were analysed with GraphPad Prism software (GraphPad). Comparisons between groups were made using Student's $t$ test (between two groups) or analysis of variance (ANOVA; multiple group comparisons), as appropriate. Statistical significance was defined as $P<0.05$.

\section{RESULTS}

\section{Tyrosine kinase(s) and PI3K are involved in $T$. forsythia invasion}

We have previously shown that protein phosphorylation and actin polymerization are required for T. forsythia entry into epithelial cells (Inagaki et al., 2006). Here we investigated the specific involvement of tyrosine kinases and PI3Ks during T. forsythia entry by using specific inhibitors. Genistein is an inhibitor of tyrosine kinase (Akiyama et al., 1987), and wortmannin and LY294002 are potent and specific inhibitors of PI3Ks (Arcaro \& Wymann, 1993; Vlahos et al., 1994). LY294002 is a highly selective inhibitor of PI3K, and does not inhibit other lipid and protein kinases (Vlahos et al., 1994). PI3K activation by phosphorylated tyrosine kinase receptors and/or adaptor proteins signals to multiple downstream effectors such as Akt to modify actin for bacterial invasion (Engelman et al., 
2006; Ireton, 2007). We observed that the T. forsythia invasion of both KB and OBA-9 cells was significantly decreased by genistein treatment in a dose-dependent manner (Fig. 1a), suggesting that T. forsythia invasion is dependent on tyrosine kinase signalling. In addition, wortmannin as well as LY294002 in a dose-dependent manner decreased the efficiency of $T$. forsythia invasion into KB or OBA- 9 cells (Fig. 1b, c). However, the reason for the reduced activity of these inhibitors in OBA-9 cells as compared with $\mathrm{KB}$ cells is not known. It is likely that in OBA-9 cells, other parallel pathways are functioning for $T$. forsythia invasion. The results nevertheless demonstrated that PI3K-dependent signalling contributes to T. forsythia invasion. In addition, activation of PI3K was confirmed by analysing for the phosphorylation of serine residue 473 in Akt (a target downstream of PI3K activation) by Western immunoblotting. The phosphorylation of Ser 473 is associated with activation of the kinase activity. Moreover, since invasion in epithelial cells has been shown to be dependent on the BspA protein (Inagaki et al., 2006), we determined whether the BspA protein could be involved in PI3K activation. For this purpose cell lysates prepared from KB or OBA-9 cells stimulated with T. forsythia, BspA or EGF as a positive control were subjected to Western immunoblotting. The results indicated that the amount of phosphoAkt in KB (Fig. 2a, b) as well as OBA-9 (Fig. 2c, d) cells increased with time initially and then returned to basal levels following challenge with $T$. forsythia. Markedly, a less robust phospho-Akt increase in OBA-9 cells as compared with $\mathrm{KB}$ cells in response to T. forsythia or BspA challenge was observed (Fig. 2). We believe that this could be due to differences in the baseline activity of PI3K between the two cell types; as shown, in comparison with KB cells, OBA-9 cells presented higher baseline levels of phospho-Akt [Fig. 2, (a) versus (c), 0 min lanes]. We predict that continued activation of PI3K in OBA-9 cells due to the presence of epidermal and other growth factors in the KGM-2 medium is a likely cause.

We observed that phospho-Akt levels in epithelial cells infected with the BspA-deficient mutant BFM571 were not significantly different from those in wild-type $T$. forsythiainfected cells (data not shown). We expected to observe reduced phospho-Akt levels in mutant-induced epithelial cells. A plausible explanation is that other bacterial components involved in invasion might also contribute to PI3K activation. Although the identity of these components is currently unknown, an earlier study has demonstrated that $T$. forsythia S-layer proteins also mediate attachment to and invasion of $\mathrm{KB}$ epithelial cells (Sabet et al., 2003). Interestingly, T. forsythia whole cells and their components have been shown to activate the pattern recognition receptor Toll-like receptor (TLR)2 (Hasebe et al., 2004; Kikkert et al., 2007; Onishi et al., 2008), and PI3K is also a downstream target of TLR2 activation. Thus, the contribution of BspA in invasion and host cell signalling in the context of the bacterial whole cell could not be discerned in this study.
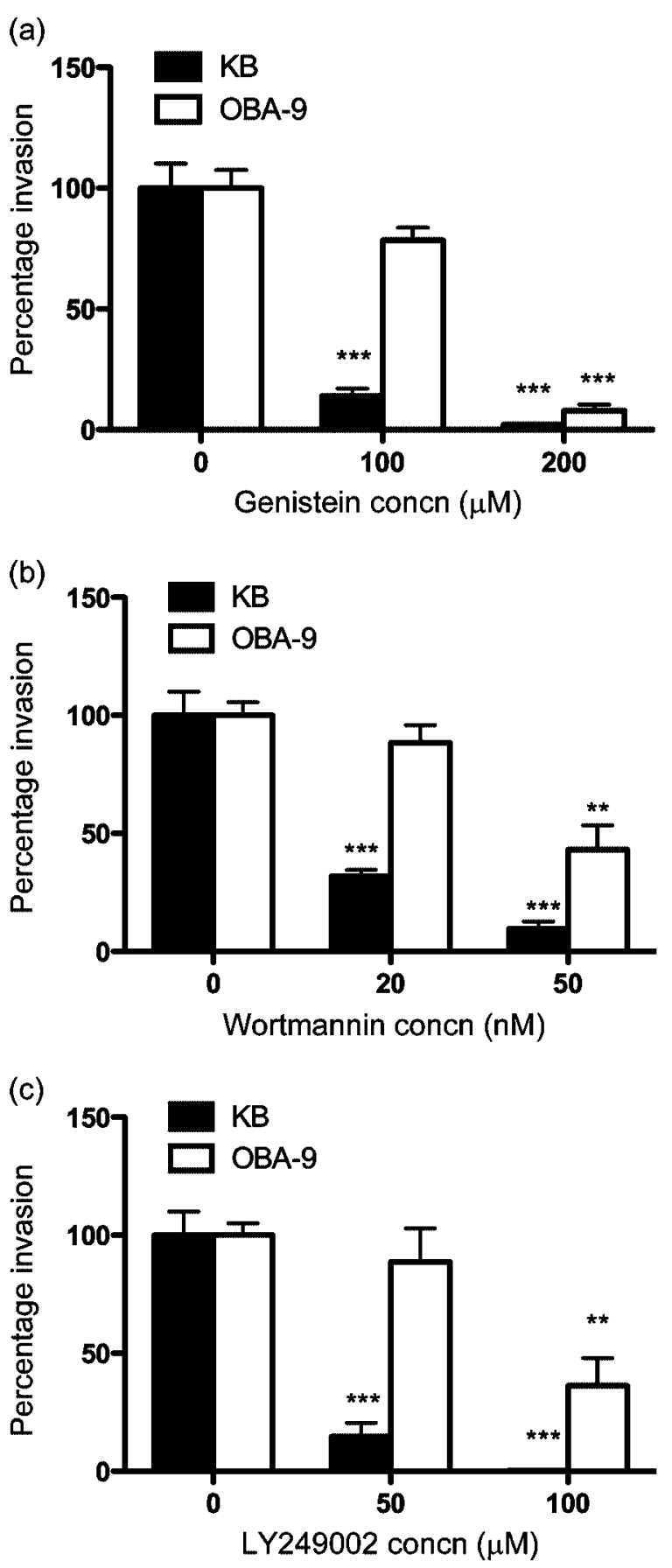

Fig. 1. Invasion assay with (a) genistein-, (b) wortmannin- and (c) LY249002-treated KB and OBA-9 cells. Values are expressed as a percentage of the bacterial invasion of non-treated cells (designated $100 \%$ ). Results are means and SDs of three independent experiments each with triplicate measurements. ** and *** indicate $P<0.01$ and $P<0.001$, respectively, relative to untreated cells.

\section{Rac1 GTPases are involved in T. forsythia invasion}

Since Rho-family GTPases link outside signals to proteins involved in cytoskeleton organization, we sought to reveal 
(a)
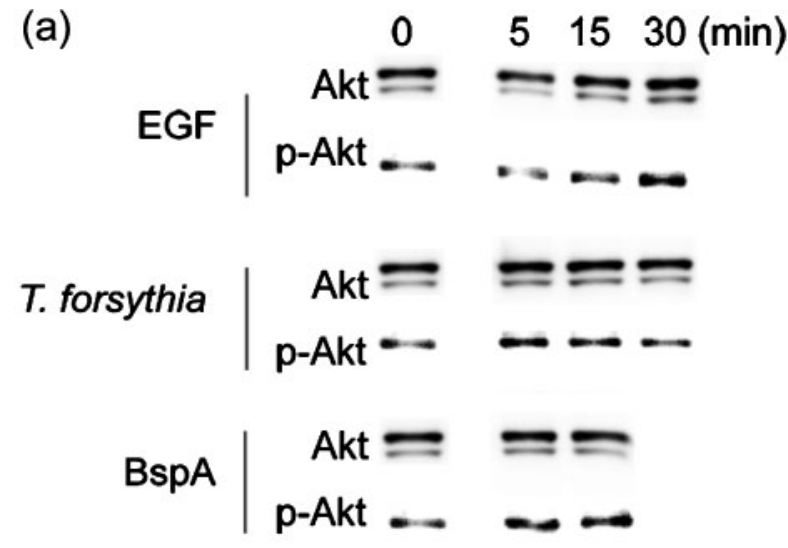

(b)

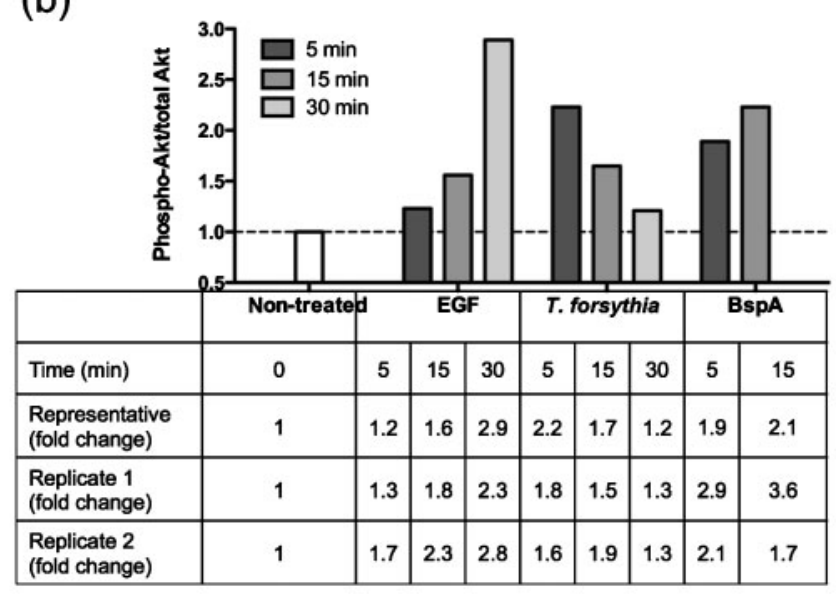

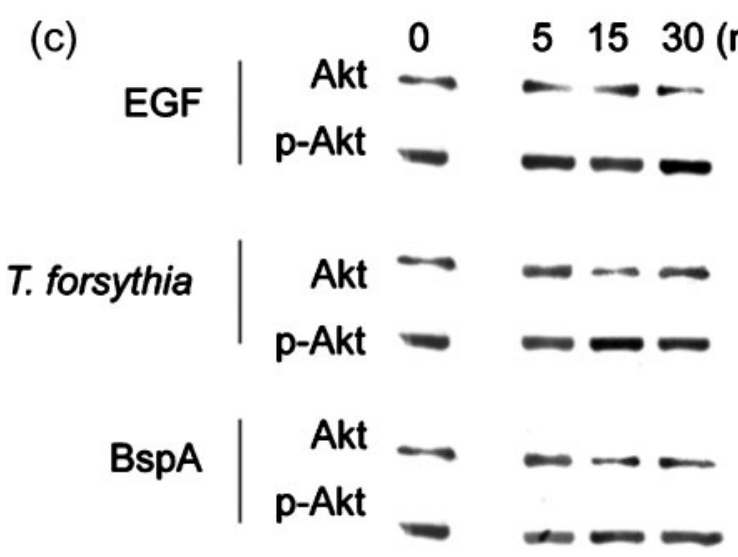

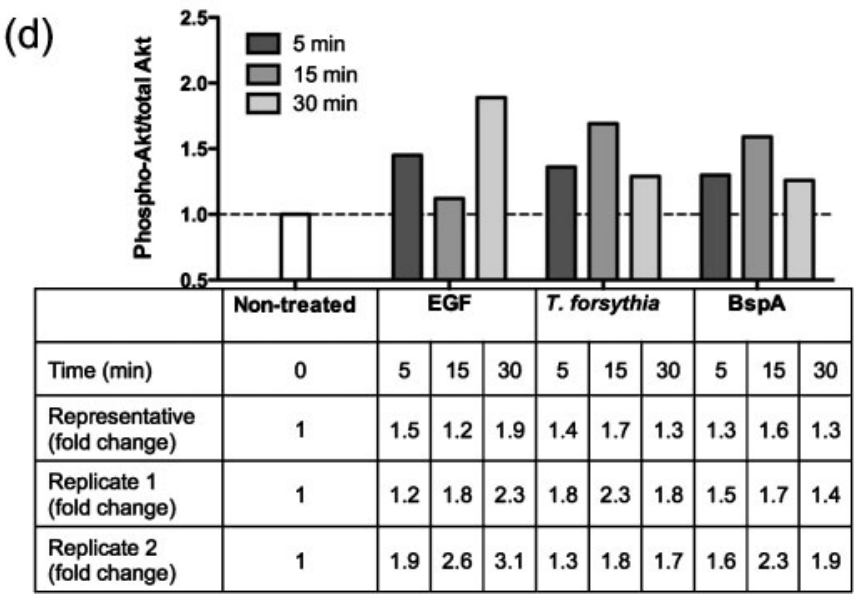

Fig. 2. Akt kinase activity in $K B(a, b)$ and OBA-9 (c, d) cells following EGF, T. forsythia or BspA treatment. (a, c) Cell extracts were analysed by Western blotting. Anti-Akt and anti-phospho-Akt antibodies were used as primary antibodies. (b, d) Densitometric analyses of immunoblots in (a) and (c) showing expression levels of phospho-Akt after various treatments relative to those of non-treated cells. Total Akt levels were used for normalization. Data were analysed with NIH ImageJ software. The tables below the bar graphs show fold changes observed for representative blots and two other independent replicates.

which of the GTPases was involved. We utilized an RNA interference (RNAi) strategy to knockdown Rac1, Cdc42 and RhoA expression in KB cells. We confirmed the knockdown efficiency by qRT-PCR, and routinely observed 80-85\% knockdown for each of the GTPase transcripts (Fig. 3a). We observed that T. forsythia invasion efficiency was decreased significantly in Racl knockdown cells as compared with that in mock- or non-specific siRNAtransfected cells (negative controls) (Fig. 3b); Cdc42 and RhoA knockdown did not decrease T. forsythia invasion efficiency significantly. The reasons for less than optimal, but significant, inhibition of $T$. forsythia invasion by Rac1 siRNA could be incomplete depletion of Racl or the presence of another isoform of Rac1 (Rac2) in these cells. Alternatively, the existence of other pathways in addition to a Rac-dependent pathway for invasion is quite likely. OBA9 cells could not be utilized for siRNA transfections, as we noticed that transfection manipulations caused cells to detach from the plates and become non-viable. The RNAi results suggested that Rac1-dependent pathways are responsible for $T$. forsythia invasion. To further confirm the role of Rac1 in KB and OBA-9, a Racl inhibitor, EHT1864 (Shutes et al., 2007), was utilized. EHT1864 is a specific high-affinity inhibitor of Racl that causes loss of bound nucleotide, inhibiting both guanine nucleotide association and Tiam1 Rac guanine nucleotide exchange factor-stimulated exchange factor activity in vitro. EHT1864 therefore places Rac in an inert and inactive state, preventing its engagement with downstream effectors (Shutes et al., 2007). In addition, we also utilized a cellpermeable form of C. botulinum C3 transferase toxin as a potent and selective inhibitor of RhoA GTPase to confirm the siRNA results. Both KB and OBA-9 cells were treated with the inhibitors for $1 \mathrm{~h}$, and before infection with $T$. forsythia for the invasion assay. The results showed that $T$. forsythia invasion was significantly decreased in EHT1864treated KB and OBA-9 cells (Fig. 4a). On the other hand, $T$. forsythia invasion was not reduced in KB or OBA-9 cells 

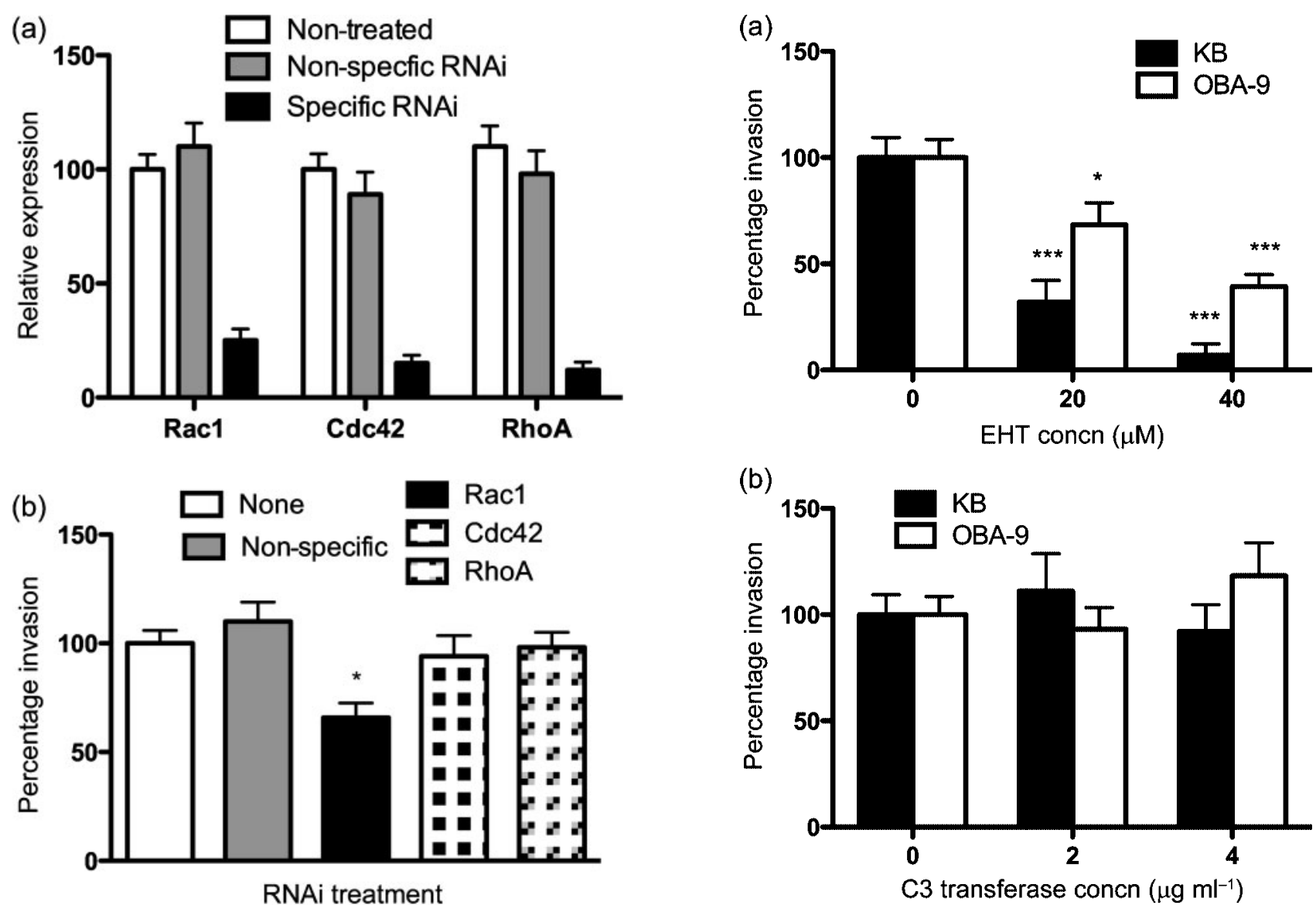

Fig. 3. (a) Relative expression of RhoA, Rac1 and Cdc42, assessed by qRT-PCR. The values were normalized with respect to GAPDH expression. The expression of each gene in non-treated KB cells was designated $100 \%$. (b) Invasion efficiency of Rac1, Cdc42 and RhoA knockdown KB cells. The number of bacteria that invaded non-treated KB cells was designated $100 \%$. Nonspecific indicates KB cells treated with negative control RNAi. Results are means and SDs of three independent experiments each with triplicate measurements. The asterisk indicates a statistically significant difference relative to untreated cells.

following treatment with C3 toxin (Fig. 4b). The activity of C3 toxin in inhibiting the cytoskeleton was confirmed by observing for the disappearance of stress-fibre formation in cells (Supplementary Fig. S1). These results demonstrated that RhoA GTPase is not responsible for $T$. forsythia invasion of epithelial cells.

\section{T. forsythia invasion requires cholesterol-rich lipid-raft domains}

The entry of many pathogens into host cells involves cholesterol- and sphingolipid-rich membrane microdomains or lipid rafts (Goluszko \& Nowicki, 2005; van der Goot \& Harder, 2001; Zaas et al., 2005). Cholesterol maintains the membrane integrity and is preferentially

Fig. 4. Invasion assay with (a) EHT1864- and (b) C3 transferasetreated KB and OBA-9 cells. Values are expressed as a percentage of bacterial invasion of non-treated KB cells (designated $100 \%)$. Error bars, SD. Asterisks indicate statistically significant differences relative to untreated cells $\left({ }^{*}, P<0.05\right.$; $\left.{ }^{\star \star *}, P<0.001\right)$.

confined to lipid rafts (Goluszko \& Nowicki, 2005). The lipid-raft domains allow receptor clustering and are rich in signalling molecules, and thus are involved in receptor signalling. Moreover, lipid rafts have been implicated in pathogen recognition and invasion. To determine whether cholesterol-enriched lipid-raft domains were involved in $T$. forsythia entry, we treated epithelial cells with the cholesterol-depleting agents $\mathrm{M} \beta \mathrm{CD}$ and nystatin. Our results showed that pretreatment of $\mathrm{KB}$ and OBA-9 cells with $\mathrm{M} \beta \mathrm{CD}$ significantly reduced the invasion of $T$. forsythia in a dose-dependent manner (Fig. 5a). Invasion by $P$. gingivalis was also significantly reduced in $\mathrm{KB}$ cells pretreated with M $\beta$ CD (Fig. 5a). P . gingivalis has been shown to utilize lipid rafts for invasion (Tamai et al., 2005), and was thus used as a positive control. Since $\mathrm{M} \beta \mathrm{CD}$ has also been shown to have pleitropic effects on other membrane processes, such as blocking clathrin-mediated endocytosis (Rodal et al., 1999; Subtil et al., 1999), we confirmed the involvement of cholesterol in $T$. forsythia entry by utilizing low dosages of 
(a)
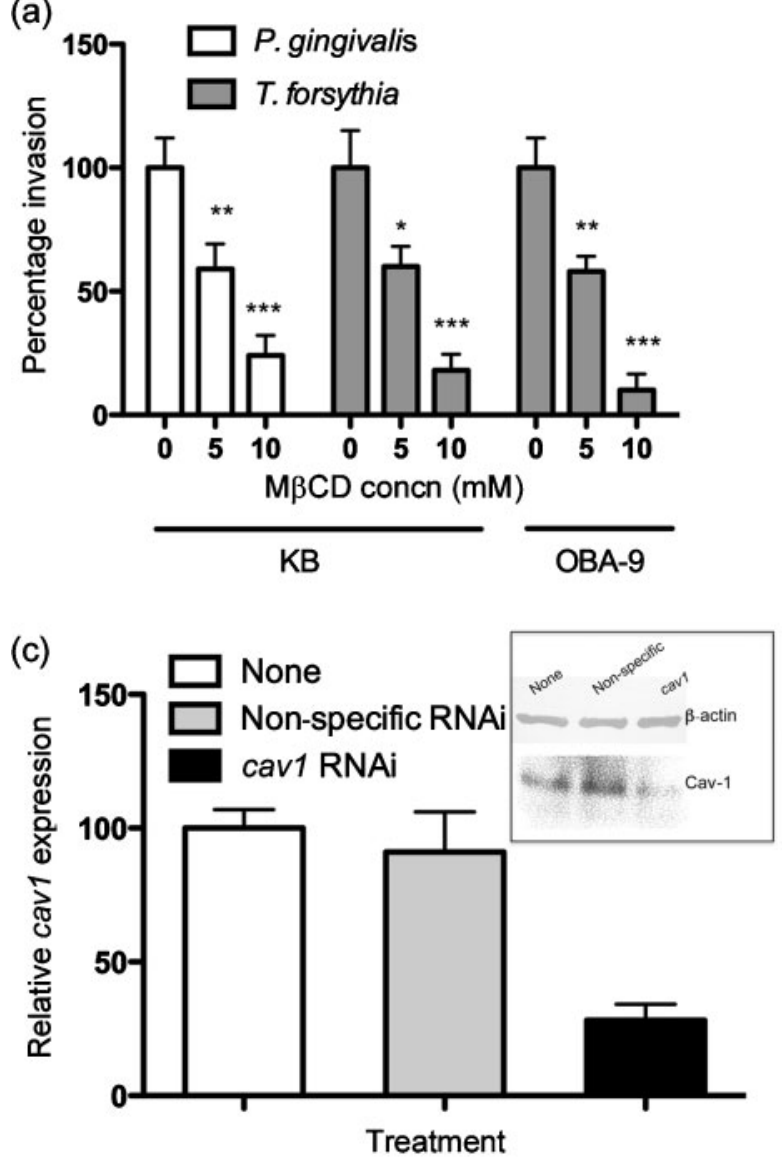

(b)
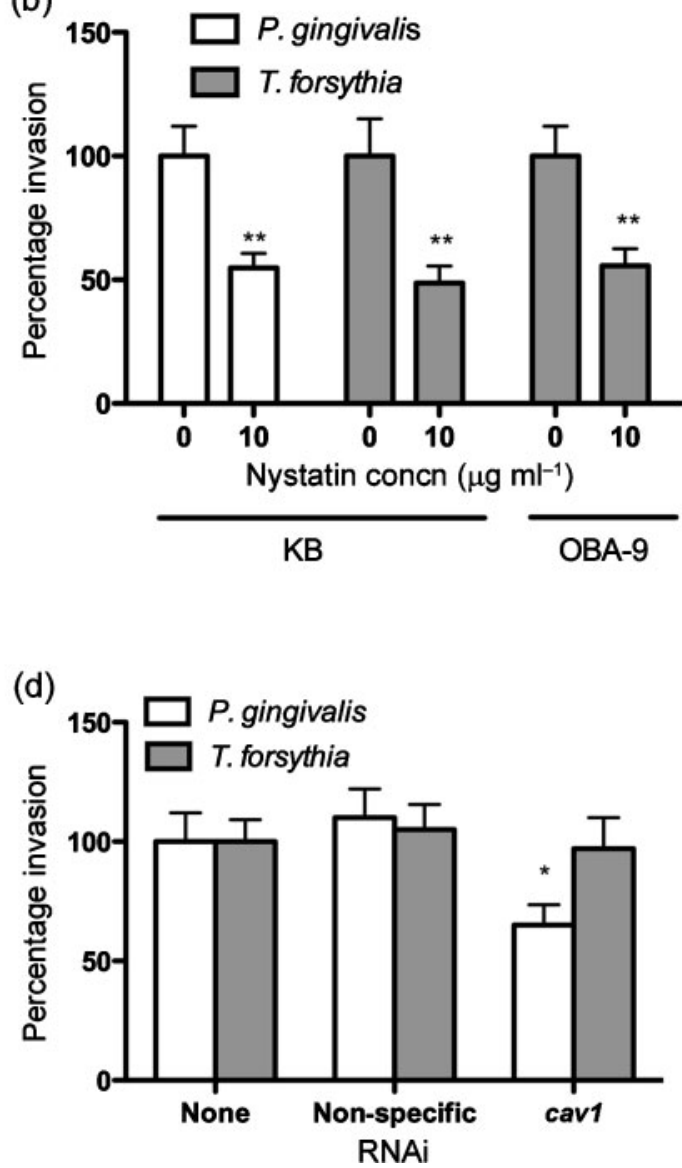

Fig. 5. $T$. forsythia invasion assay with (a) $M \beta C D$ - and (b) nystatin-treated $K B$ and OBA- 9 cells. The number of bacteria that invaded non-treated KB cells was designated $100 \%$. Results are means and SEMs of three independent experiments each with triplicate measurements. Asterisks indicate statistically significant differences relative to untreated cells $\left({ }^{\star}, P<0.05 ;{ }^{\star \star}, P<0.01\right.$; $\star \star \star, P<0.001)$. (c) Relative expression of Cav-1 was assessed by qRT-PCR and Western immunoblotting. For qRT-PCR, the values were normalized with respect to $\beta$-actin expression. The Cav-1 expression of non-treated KB cells was designated $100 \%$. (d) T. forsythia and $P$. gingivalis invasion efficiency in Cav-1 knockdown KB cells. The number of bacteria that invaded non-treated KB cells was designated $100 \%$. Results are means and SEMs of three independent experiments each with triplicate measurements. *, $P<0.05$ relative to untreated cells.

nystatin. Nystatin is a sterol-binding reagent that removes cholesterol from the plasma membrane but does not affect clathrin-coated pits, actin cables or other submembranous structures (Rothberg et al., 1992). As shown in Fig. 5(b), pretreatment of $\mathrm{KB}$ as well as OBA-9 cells with a low concentration of nystatin reduced $T$. forsythia invasion significantly as compared with non-treated cells. Invasion by P. gingivalis, which requires lipid rafts (Furuta et al., 2009), was likewise blocked in $\mathrm{KB}$ cells following nystatin pretreatment (Fig. 5b). These results are consistent with the notion that the cholesterol in lipid rafts is required for internalization of $T$. forsythia.

Lipid rafts are involved in the formation of specialized flask-shaped structures called caveolae that possess a versatile endocytic capacity for bacteria and viruses and even large parasites (Duncan et al., 2002; van der Goot \&
Harder, 2001). To further confirm the role of caveolae in $T$. forsythia entry we utilized RNAi-treated Cav-1-deficient KB cells. Cav-1, encoded by the cav1 gene, is a cholesterolbinding membrane protein and is the major protein of caveolae (Parton \& Simons, 2007). The expression of Cav-1 based on transcript and protein levels was more than $80 \%$ reduced in specfic RNAi-treated cells compared with either mock- or non-specific RNAi-treated cells (Fig. 5c). The invasion efficiency of $T$. forsythia in Cav-1 knockdown cells indicated no significant difference from cells expressing normal levels of Cav-1 (Fig. 5d). However, as expected (Tamai et al., 2005), the invasion efficiency of $P$. gingivalis used as a positive control decreased following Cav-1 knockdown (Fig. 5d). Our data demonstrated that caveolae do not mediate invasion of $T$. forsythia in epithelial cells. The results also indicate that $T$. forsythia entry is mediated via lipid rafts lacking caveolae. 


\section{T. forsythia requires the clathrin-mediated endocytic pathway for entry}

Since recent studies have demonstrated that some bacteria utilize clathrin-dependent entry as a general pathway for invasion of host cells (Veiga et al., 2007), we sought to determine whether that was also the case for T. forsythia. To reveal the involvement of clathrin-coated vesicle transportation during invasion by $T$. forsythia, we used RNAi to knockdown clathrin expression in cells. In addition, we utilized the clathrin inhibitors MDC and chlorpromazine to block clathrin function in epithelial cells prior to T. forsythia infection. MDC (Schlegel et al., 1982) and chlorpromazine (Wang et al., 1993) block receptor-mediated endocytosis via clathrin-coated pits. Clathrin forms a triskelion shape which is composed of three CLTCs and three light chains. We used siRNA to target CLTC, and calculated the invasion efficiency of $T$. forsythia. The efficiency of CLTC gene expression measured by qRT-PCR as well as protein levels measured by Western immunoblotting indicated a more than $80 \%$ reduction in cells treated with clathrin-specific siRNA compared with that in non-treated or non-specific siRNAtreated cells (Fig. 6a). Furthermore, the clathrin knockdown was assessed by determining the invasion efficiency of fluorescently labelled transferrin, which utilizes clathrincoated pits after binding to its receptor. The results confirmed that clathrin knockdown significantly reduced transferrin uptake by $\mathrm{KB}$ cells in comparison with $\mathrm{KB}$ cells expressing normal levels of clathrin (data not shown). Under similar conditions of clathrin knockdown, the invasion efficiency of T. forsythia was significantly reduced (Fig. 6b). We were not able to completely block clathrin-mediated invasion by $T$. forsythia with clathrin RNAi. This is likely due to incomplete blocking of clathrin expression in cells by the RNAi approach. The RNAi results were corroborated with the inhibitors MDC and chlorpromazine, both of which (a)

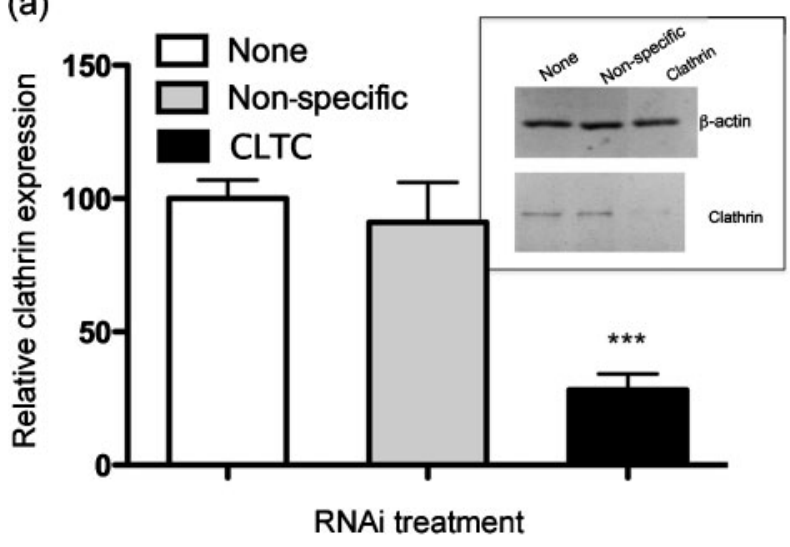

(c)

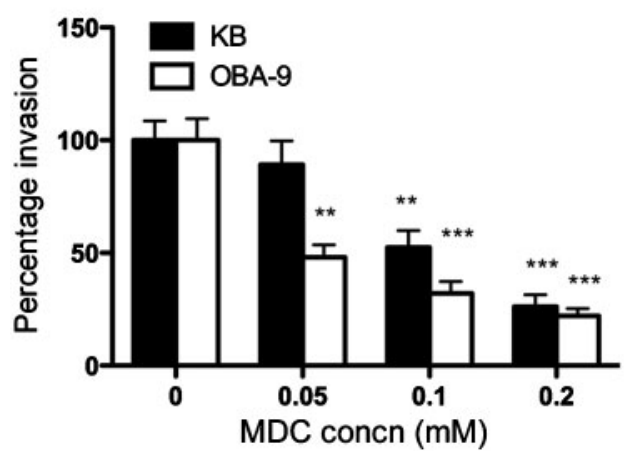

(b)

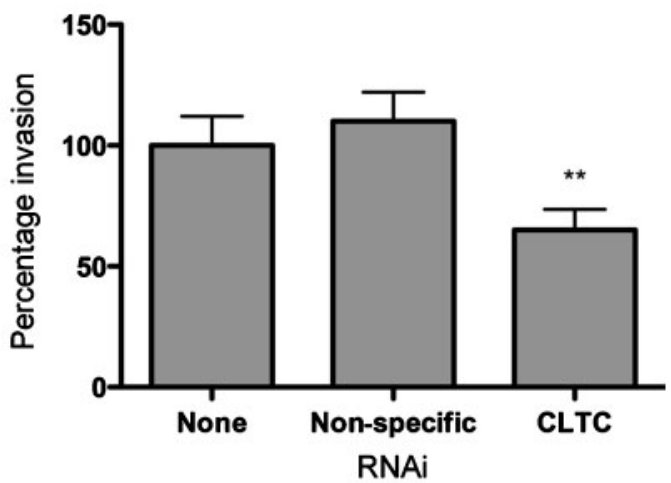

(d)

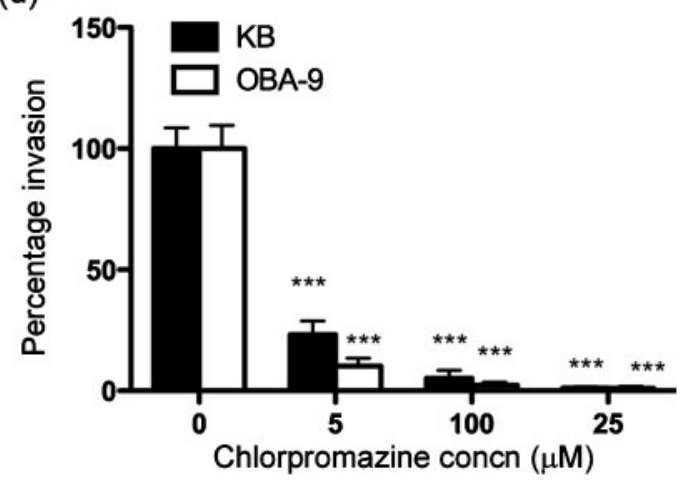

Fig. 6. (a) Relative expression of CLTC assessed by qRT-PCR and Western immunoblotting. For qRT-PCR, values were normalized with respect to $\beta$-actin expression. The CLTC expression of non-treated KB cells was designated $100 \%$ $\left({ }^{\star \star \star}, P<0.001\right)$. (b) T. forsythia infection efficiency in CLTC knockdown KB cells. The number of bacteria that invaded nontreated KB cells was designated $100 \%$. Results are means and SEMS of three independent experiments each with triplicate measurements. Asterisks indicate statistically significant differences relative to untreated cells $\left({ }^{* *}, P<0.01\right)$. (c, d) $T$. forsythia invasion assay with MDC- and chloropromazine-treated KB and OBA-9 cells. The number of bacteria that invaded non-treated KB or OBA- 9 cells was designated $100 \%$. Results are means and SDs of three independent experiments each with triplicate measurements. Asterisks indicate statistically significant differences relative to untreated cells $\left({ }^{\star \star}, P<0.01 ;{ }^{\star \star *}, P<0.001\right)$. 
dose-dependently inhibited $T$. forsythia invasion of KB as well as OBA-9 cells (Fig. 6c, d).

\section{DISCUSSION}

The data presented in this study show that signal transduction involving activation of host PI3K, Akt and the Rho GTPase Racl is involved in T. forsythia entry into epithelial cells. In addition, the results show that $T$. forsythia BspA protein activates PI3K. Our study also reveals an important role for the endocytic protein clathrin in bacterial uptake. Finally, while lipid rafts are involved in invasion, lipid raftassociated caveolae containing Cav-1 protein are not involved in the entry process.

While we demonstrated roles for PI3K and Rac1-dependent signalling during $T$. forsythia invasion, our studies also indicated that other signalling pathways might be involved. Moreover, the pathways might be host cell-specific. This notion is based on the fact that inhibiting PI3K activity was less effective in blocking the invasion of $T$. forsythia in oral epithelial OBA-9 cells compared with cervical epithelial $\mathrm{KB}$ cells. Likewise, Rac1 knockdown was only partially, though significantly, effective in blocking bacterial invasion in $\mathrm{KB}$ cells. With regard to PI3K activation by whole cells and BspA protein, PI3K-mediated signalling has been implicated in the invasion of many bacteria, such as Yersinia, Pseudomonas aeruginosa and Listeria (Ireton et al., 1996; Kannan et al., 2008; Kierbel et al., 2005; Schulte et al., 1998). PI3K activation controls the levels of the lipid messengers phosphatidylinositol 3,4,5-trisphosphate and phosphatidylinositol 3,4-bisphosphate, which in turn can regulate the activity of downstream effectors (GTPase-activating proteins and guanine nucleotide exchange factors) involved in activation of Rho GTPase (Vanhaesebroeck et al., 2010). Activated Rho GTPases then recruit downstream effectors such as Arp2/3 and WAVE/ WASP complexes, which in turn promote actin polymerization (Ridley, 2006). Actin polymerization mediated by Rho GTPases plays critical roles in many cellular events, including cell-cell and cell-substrate adhesion, cell motility, and the uptake of foreign particles via phagocytosis. Since actin polymerization is also critical for $T$. forsythia entry into epithelial cells (Inagaki et al., 2006), it is reasonable to speculate that PI3K-Racl activation would induce actin cytoskeletal changes by recruiting Arp $2 / 3$ and WAVE/WASP during entry. We plan to test this possibility in our future studies.

It is not known at the moment to which epithelial receptor BspA binds and how that leads to PI3K and Racl activation to cause T. forsythia invasion. The predicted structure of the BspA protein indicates that the protein contains LRR and bacterial immunoglobulin-like domains (Big_2) (Sharma, 2010). These domains have been implicated in protein-protein interactions and thus may be involved in receptor binding. In case of Listeria monocytogenes, two different LRRs containing internalin proteins InlA and InlB bind distinctly different receptors to trigger cellular signalling and cause bacterial entry (Dokainish et al.,
2007; Mostowy et al., 2009), and in Prevotella intermedia, the LRR protein AdpC has recently been shown to mediate invasion in a cell-type-specific manner (Iyer et al., 2010).

The ability of the cholesterol-sequestering agents $\mathrm{M} \beta \mathrm{CD}$ and nystatin to inhibit $T$. forsythia invasion suggests that membrane lipid rafts are involved in entry. Lipid rafts are membrane microdomains highly enriched in cholesterol, sphingolipids, glycolipids and glycosylphosphatidylinositolanchored receptors. Some, but not all, lipid rafts contain Cav-1 protein (Brown \& London, 2000). Mechanistically, membrane receptors cluster in lipid-raft domains following micro-organism engagement (Mañes et al., 2003; Riethmüller et al., 2006). The clustering of these receptors triggers sorting signals for selective endocytosis through GTPase-regulated actin polymerization (Lafont et al., 2004). For example, in the case of L. monocytogenes, depletion of cholesterol before infection with InlA-expressing Listeria or before incubation with InlA-coated microbeads reduces clustering of E-cadherin and affects the initial adhesion to eukaryotic cells (Seveau et al., 2004). Since the BspA receptor has not been identified, currently it is difficult to predict whether receptor clustering would be a prerequisite for entry.

Recent studies have provided evidence for the clathrinbased entry of a number of pathogens (Cossart \& Sansonetti, 2004; Veiga \& Cossart, 2006; Veiga et al., 2007). This is in contrast to the previously imputed role for clathrin in the internalization or 'endocytosis' of smaller molecular cargo in the range of 30-150 nm (Ehrlich et al., 2004). Our findings that reveal both lipid raft- and clathrin-dependent entry of $T$. forsythia are intriguing. To our knowledge, clathrin-based (unlike caveolin-based) endocytosis is independent of lipid rafts. Therefore, it is plausible that the lipid raft- and clathrin-mediated pathways are independent and play functionally redundant roles in T. forsythia entry. Alternatively, the possibility also exists that rafts and clathrin are both required for signal transduction related to bacterial entry. This is very likely in relation to rafts, as they do play roles in signal transduction (Golub et al., 2004). In fact, rafts have been shown to be required for Rac activation for $L$. monocytogenes entry (Seveau et al., 2007). A similar scenario for clathrin, though less documented, is not improbable. Recent evidence has indicated that clathrin scaffolds play roles in localization and/or the activities of protein and lipid kinases (Mangmool et al., 2006).

In summary, our results illustrate for the first time the key signalling events of $T$. forsythia invasion into epithelial cells. We show that the bacterial entry process is dependent on the cooperation and activation of the Rho-family GTPase Rac1 and PI3K. Furthermore, the entry process requires lipid-raft microdomains and the endocytic protein clathrin.

\section{ACKNOWLEDGEMENTS}

We thank Keith Ireton for his helpful suggestions throughout the course of this study. This study was supported by the National 
Institute of Dental and Craniofacial Research (NIDCR) (grants DE014749 and DE019424).

\section{REFERENCES}

Akiyama, T., Ishida, J., Nakagawa, S., Ogawara, H., Watanabe, S., Itoh, N., Shibuya, M. \& Fukami, Y. (1987). Genistein, a specific inhibitor of tyrosine-specific protein kinases. J Biol Chem 262, 55925595.

Arcaro, A. \& Wymann, M. P. (1993). Wortmannin is a potent phosphatidylinositol 3-kinase inhibitor: the role of phosphatidylinositol 3,4,5-trisphosphate in neutrophil responses. Biochem J 296, 297 301.

Brown, D. A. \& London, E. (2000). Structure and function of sphingolipid- and cholesterol-rich membrane rafts. J Biol Chem 275, 17221-17224.

Colombo, A. V., da Silva, C. M., Haffajee, A. \& Colombo, A. P. (2007). Identification of intracellular oral species within human crevicular epithelial cells from subjects with chronic periodontitis by fluorescence in situ hybridization. J Periodontal Res 42, 236-243.

Cossart, P. \& Sansonetti, P. J. (2004). Bacterial invasion: the paradigms of enteroinvasive pathogens. Science 304, 242-248.

Dokainish, H., Gavicherla, B., Shen, Y. \& Ireton, K. (2007). The carboxyl-terminal SH3 domain of the mammalian adaptor CrkII promotes internalization of Listeria monocytogenes through activation of host phosphoinositide 3-kinase. Cell Microbiol 9, 2497-2516.

Duncan, M. J., Shin, J. S. \& Abraham, S. N. (2002). Microbial entry through caveolae: variations on a theme. Cell Microbiol 4, 783-791.

Ehrlich, M., Boll, W., Van Oijen, A., Hariharan, R., Chandran, K., Nibert, M. L. \& Kirchhausen, T. (2004). Endocytosis by random initiation and stabilization of clathrin-coated pits. Cell 118, 591-605.

Engelman, J. A., Luo, J. \& Cantley, L. C. (2006). The evolution of phosphatidylinositol 3-kinases as regulators of growth and metabolism. Nat Rev Genet 7, 606-619.

Furuta, N., Tsuda, K., Omori, H., Yoshimori, T., Yoshimura, F. \& Amano, A. (2009). Porphyromonas gingivalis outer membrane vesicles enter human epithelial cells via an endocytic pathway and are sorted to lysosomal compartments. Infect Immun 77, 4187-4196.

Golub, T., Wacha, S. \& Caroni, P. (2004). Spatial and temporal control of signaling through lipid rafts. Curr Opin Neurobiol 14, 542550 .

Goluszko, P. \& Nowicki, B. (2005). Membrane cholesterol: a crucial molecule affecting interactions of microbial pathogens with mammalian cells. Infect Immun 73, 7791-7796.

Han, Y. W., Shi, W., Huang, G. T., Kinder Haake, S., Park, N. H., Kuramitsu, H. \& Genco, R. J. (2000). Interactions between periodontal bacteria and human oral epithelial cells: Fusobacterium nucleatum adheres to and invades epithelial cells. Infect Immun 68, 3140-3146.

Hasebe, A., Yoshimura, A., Into, T., Kataoka, H., Tanaka, S., Arakawa, S., Ishikura, H., Golenbock, D. T., Sugaya, T. \& other authors (2004). Biological activities of Bacteroides forsythus lipoproteins and their possible pathological roles in periodontal disease. Infect Immun 72, 1318-1325.

Honma, K., Kuramitsu, H. K., Genco, R. J. \& Sharma, A. (2001). Development of a gene inactivation system for Bacteroides forsythus: construction and characterization of a BspA mutant. Infect Immun 69, 4686-4690.

Honma, K., Mishima, E. \& Sharma, A. (2011). Role of Tannerella forsythia $\mathrm{NanH}$ sialidase in epithelial cell attachment. Infect Immun 79, 393-401.
Inagaki, S., Onishi, S., Kuramitsu, H. K. \& Sharma, A. (2006). Porphyromonas gingivalis vesicles enhance attachment, and the leucine-rich repeat BspA protein is required for invasion of epithelial cells by "Tannerella forsythia". Infect Immun 74, 5023-5028.

Ireton, K. (2007). Entry of the bacterial pathogen Listeria monocytogenes into mammalian cells. Cell Microbiol 9, 1365-1375.

Ireton, K., Payrastre, B., Chap, H., Ogawa, W., Sakaue, H., Kasuga, M. \& Cossart, P. (1996). A role for phosphoinositide 3-kinase in bacterial invasion. Science 274, 780-782.

Iyer, D., Anaya-Bergman, C., Jones, K., Yanamandra, S., Sengupta, D., Miyazaki, H. \& Lewis, J. P. (2010). AdpC is a Prevotella intermedia 17 leucine-rich repeat internalin-like protein. Infect Immun 78, 23852396.

Kannan, S., Audet, A., Huang, H., Chen, L. J. \& Wu, M. (2008). Cholesterol-rich membrane rafts and Lyn are involved in phagocytosis during Pseudomonas aeruginosa infection. J Immunol 180, 23962408.

Kierbel, A., Gassama-Diagne, A., Mostov, K. \& Engel, J. N. (2005). The phosphoinositol-3-kinase-protein kinase B/Akt pathway is critical for Pseudomonas aeruginosa strain PAK internalization. Mol Biol Cell 16, 2577-2585.

Kikkert, R., Laine, M. L., Aarden, L. A. \& van Winkelhoff, A. J. (2007). Activation of Toll-like receptors 2 and 4 by Gram-negative periodontal bacteria. Oral Microbiol Immunol 22, 145-151.

Kirschbaum, M., Schultze-Mosgau, S., Pfister, W. \& Eick, S. (2010). Mixture of periodontopathogenic bacteria influences interaction with KB cells. Anaerobe 16, 461-468.

Lafont, F., Abrami, L. \& van der Goot, F. G. (2004). Bacterial subversion of lipid rafts. Curr Opin Microbiol 7, 4-10.

Mañes, S., del Real, G. \& Martínez-A, C. (2003). Pathogens: raft hijackers. Nat Rev Immunol 3, 557-568.

Mangmool, S., Haga, T., Kobayashi, H., Kim, K. M., Nakata, H., Nishida, M. \& Kurose, H. (2006). Clathrin required for phosphorylation and internalization of $\beta_{2}$-adrenergic receptor by $\mathrm{G}$ proteincoupled receptor kinase 2 (GRK2). J Biol Chem 281, 31940-31949.

Mostowy, S., Danckaert, A., Tham, T. N., Machu, C., Guadagnini, S., Pizarro-Cerdá, J. \& Cossart, P. (2009). Septin 11 restricts InlBmediated invasion by Listeria. J Biol Chem 284, 11613-11621.

Onishi, S., Honma, K., Liang, S., Stathopoulou, P., Kinane, D., Hajishengallis, G. \& Sharma, A. (2008). Toll-like receptor 2-mediated interleukin-8 expression in gingival epithelial cells by the Tannerella forsythia leucine-rich repeat protein BspA. Infect Immun 76, 198205.

Parton, R. G. \& Simons, K. (2007). The multiple faces of caveolae. Nat Rev Mol Cell Biol 8, 185-194.

Ridley, A. J. (2006). Rho GTPases and actin dynamics in membrane protrusions and vesicle trafficking. Trends Cell Biol 16, 522-529.

Riethmüller, J., Riehle, A., Grassmé, H. \& Gulbins, E. (2006). Membrane rafts in host-pathogen interactions. Biochim Biophys Acta 1758, 2139-2147.

Rodal, S. K., Skretting, G., Garred, O., Vilhardt, F., van Deurs, B. \& Sandvig, K. (1999). Extraction of cholesterol with methyl-betacyclodextrin perturbs formation of clathrin-coated endocytic vesicles. Mol Biol Cell 10, 961-974.

Rothberg, K. G., Heuser, J. E., Donzell, W. C., Ying, Y. S., Glenney, J. R. \& Anderson, R. G. (1992). Caveolin, a protein component of caveolae membrane coats. Cell 68, 673-682.

Rudney, J. D., Chen, R. \& Sedgewick, G. J. (2005). Actinobacillus actinomycetemcomitans, Porphyromonas gingivalis, and Tannerella forsythensis are components of a polymicrobial intracellular flora within human buccal cells. J Dent Res 84, 59-63. 
Sabet, M., Lee, S. W., Nauman, R. K., Sims, T. \& Um, H. S. (2003). The surface (S-) layer is a virulence factor of Bacteroides forsythus. Microbiology 149, 3617-3627.

Schlegel, R., Dickson, R. B., Willingham, M. C. \& Pastan, I. H. (1982). Amantadine and dansylcadaverine inhibit vesicular stomatitis virus uptake and receptor-mediated endocytosis of alpha 2-macroglobulin. Proc Natl Acad Sci U S A 79, 2291-2295.

Schulte, R., Zumbihl, R., Kampik, D., Fauconnier, A. \& Autenrieth, I. B. (1998). Wortmannin blocks Yersinia invasin-triggered internalization, but not interleukin-8 production by epithelial cells. Med Microbiol Immunol (Berl) 187, 53-60.

Seveau, S., Bierne, H., Giroux, S., Prévost, M. C. \& Cossart, P. (2004). Role of lipid rafts in E-cadherin- and HGF-R/Met-mediated entry of Listeria monocytogenes into host cells. J Cell Biol 166, 743753.

Seveau, S., Tham, T. N., Payrastre, B., Hoppe, A. D., Swanson, J. A. \& Cossart, P. (2007). A FRET analysis to unravel the role of cholesterol in Racl and PI 3-kinase activation in the InlB/Met signalling pathway. Cell Microbiol 9, 790-803.

Sharma, A. (2010). Virulence mechanisms of Tannerella forsythia. Periodontol 2000 54, 106-116.

Shutes, A., Onesto, C., Picard, V., Leblond, B., Schweighoffer, F. \& Der, C. J. (2007). Specificity and mechanism of action of EHT 1864, a novel small molecule inhibitor of Rac family small GTPases. J Biol Chem 282, 35666-35678.

Subtil, A., Gaidarov, I., Kobylarz, K., Lampson, M. A., Keen, J. H. \& McGraw, T. E. (1999). Acute cholesterol depletion inhibits clathrincoated pit budding. Proc Natl Acad Sci U S A 96, 6775-6780.
Tamai, R., Asai, Y. \& Ogawa, T. (2005). Requirement for intercellular adhesion molecule 1 and caveolae in invasion of human oral epithelial cells by Porphyromonas gingivalis. Infect Immun 73, 6290-6298.

Tanner, A. C. \& Izard, J. (2006). Tannerella forsythia, a periodontal pathogen entering the genomic era. Periodontol 2000 42, 88-113.

van der Goot, F. G. \& Harder, T. (2001). Raft membrane domains: from a liquid-ordered membrane phase to a site of pathogen attack. Semin Immunol 13, 89-97.

Vanhaesebroeck, B., Guillermet-Guibert, J., Graupera, M. \& Bilanges, B. (2010). The emerging mechanisms of isoform-specific PI3K signalling. Nat Rev Mol Cell Biol 11, 329-341.

Veiga, E. \& Cossart, P. (2006). The role of clathrin-dependent endocytosis in bacterial internalization. Trends Cell Biol 16, 499-504.

Veiga, E., Guttman, J. A., Bonazzi, M., Boucrot, E., Toledo-Arana, A., Lin, A. E., Enninga, J., Pizarro-Cerdá, J., Finlay, B. B. \& other authors (2007). Invasive and adherent bacterial pathogens co-opt host clathrin for infection. Cell Host Microbe 2, 340-351.

Vlahos, C. J., Matter, W. F., Hui, K. Y. \& Brown, R. F. (1994). A specific inhibitor of phosphatidylinositol 3-kinase, 2-(4-morpholinyl)-8-phenyl4H-1-benzopyran-4-one (LY294002). J Biol Chem 269, 5241-5248.

Wang, L. H., Rothberg, K. G. \& Anderson, R. G. (1993). Mis-assembly of clathrin lattices on endosomes reveals a regulatory switch for coated pit formation. J Cell Biol 123, 1107-1117.

Zaas, D. W., Duncan, M., Rae Wright, J. \& Abraham, S. N. (2005). The role of lipid rafts in the pathogenesis of bacterial infections. Biochim Biophys Acta 1746, 305-313.

Edited by: W. Crielaard 